\title{
Personal Comfort Node: Prototyping of a Comfort Sensor for HVAC Control
}

\author{
Joseph Rosenberger, Austin Coffman, \& Prabir Barooah \\ University of Florida
}

Faculty Mentor: Prabir Barooah, Department of Mechanical and Aerospace Engineering

\begin{abstract}
This paper presents the design of a Personal Comfort Node (PCN), a platform that senses environmental variables such as temperature and allows users to submit feedback on comfort. It is meant to be part of an indoor climate control system that can provide personalized occupant comfort in commercial buildings. The paper describes the lessons learned during the design and prototyping of an individual node. The hardware components are of commercial-off-the-shelf variety and the software design is based on open source tools that are freely available, so that it is possible to replicate the device at low cost. The goal of this article is to aid such replication. This article focuses on the development of the sensing platform. The design and testing of a network of these devices, along with their use in real-time control of indoor climate, will be reported in future work. The eventual use of the data from the PCN for control is not a topic of this paper

Keywords: HVAC control system, comfort model, Arduino
\end{abstract}

\section{Introduction}

The purpose of building HVAC systems is to provide a comfortable and healthy indoor environment. Although HVAC equipment and their control systems have grown in sophistication over the years, comfort for all occupants in a large building is still an unrealized goal. This occurs due to a number of factors, in spite of the best efforts of HVAC design engineers and control vendors. A key factor is that there is no comfort sensor, since a host of factors affect a person's perception of comfort, and not all of them are measurable. Comfort levels cannot be determined by environmental sensing alone; user input is also required. Unfortunately, the range of environmental variables that are considered comfortable by a person varies among individuals, which makes it hard to keep everyone equally happy. Since a person is the best judge for what is comfortable for that person, a climate control system should ideally involve the 
occupant in the climate control loop. The key challenge is to get useful information and yet not be disruptive.

The Personal Comfort Node (PCN) described in this report is motivated by this state of affairs. Each PCN device consists of a number of sensors that measure environmental variables, and a touch screen through which occupants can provide feedback about their perceived comfort. Figure 1 shows a prototype of a PCN device. This data can be used to learn a comfort model of the person that interacts with the PCN device. It is envisioned that each person will have one PCN device in their workspace that is assigned to them. In the simplest case a comfort model is simply the range of values the environmental variables can take so that the person is comfortable. A control system can then use the model to manipulate the environment through the HVAC system in the building to bring it to within that range if possible. It will do this by adjusting the air flow that the HVAC system outputs to bring the temperature of each room down or up. The temperature of each room will correspond to the preferences of the occupant of that room. Other factors that contribute to an occupants comfort preferences will be considered to when changing the temperature. These include humidity, $\mathrm{CO} 2$ levels, time of day, and more. If there are multiple occupants in one space, and they have sufficiently distinct preferences, the control system may have to strike some sort of compromise. Ultimately the goal is to create more comfort in occupants based on their preferences, which in turn leads to greater productivity. The details of how the data and the models will be used is beyond this paper.

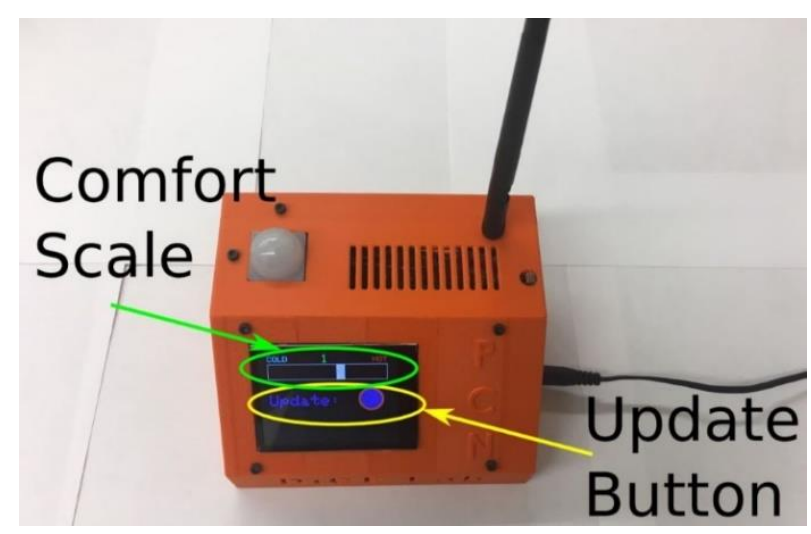

Figure 1. A powered on PCN device. The comfort scale and update button are shown. 


\section{Prior Work}

Devices similar to the PCN have been created with similar intent. Feldmeier and Paradiso (2010) created nodes wearable on the wrist. The node consists of a few environmental sensors, user input through three options (cold, neutral, or hot), wireless communication, and location tracking. Smartphone apps have also been used to take in user input, with external sensors for collecting environmental data (Gupta et al., 2016; Jazizadeh et al., 2014). This comes with an additional problem of mapping the smartphone location to the external sensors. Pitt et al., (2013) created a wired network of 17 devices that also provides three user inputted options.

The goal is to make the design of the PCN device and the network publicly available so that others can reproduce such a system. In this aspect the goal is similar to (Ali et al., 2016). There are many similarities between the system described in (Ali et al., 2016) and the PCN. The system described in (Ali et al., 2016) aims to provide an inexpensive, open source device that utilizes numerous sensors with the ultimate goal of collecting data for building automation and control. The main differences are the presence of the touch screen on the PCN, which requires significant care in packaging to avoid heating issues, and wireless communication of data to a cloud server for the PCN. This paper focuses on the design of an individual PCN. The development of the multi-node wireless network (including server side routines and database designs) is beyond the scope of this paper and will be reported in future work. However, a range test of an individual $\mathrm{PCN}$ in relation to the base station is reported in this paper.

\section{The PCN Device}

Recall, the purpose of each PCN device is to give occupants the ability to communicate their level of comfort to the building climate control system. In order to achieve this purpose the PCN device is equipped with:

1. Capacitive touch screen

2. Radio and antenna

3. Sensors

4. Arduino Mega

5. 9V 1A DC power supply

The cost of all the components is outlined in Table 1 . The total cost of the device came out to be $\$ 284.66$. 


\section{Main Components}

\begin{tabular}{lccc}
\multicolumn{4}{l}{ Table 1. PCN Device Cost Analysis } \\
\hline Parts & Quantity & Unit Price $(\$)$ & Total Price (\$) \\
\hline VOC sensor & 1 & 11.96 & 11.96 \\
CO2 sensor & 1 & 65 & 65 \\
Motion sensor & 1 & 14.99 & 14.99 \\
Light sensor & 1 & 1.20 & 1.20 \\
Humidity sensor & 1 & 16.11 & 16.11 \\
Temperature sensor & 1 & 3.56 & 3.56 \\
Housing material & 1 & 42 & 42 \\
Threaded hex standoff & 17 & 0.23 & 3.91 \\
Socket head screw & 17 & 0.085 & 1.45 \\
Capacitive touch screen & 1 & 35.96 & 35.96 \\
Arduino Mega 2560 R3 & 1 & 22.99 & 22.99 \\
XBEE-Pro Zigbee & 1 & 28.5 & 28.5 \\
RPSMA antenna & 1 & 7.16 & 7.16 \\
XBEE breakout board & 1 & 2.51 & 2.51 \\
XBEE header pins & 2 & 0.9 & 1.8 \\
DC barrel jack adapter & 1 & 2.66 & 2.66 \\
Voltage supply & 1 & 8 & 8 \\
PCB large & 1 & 13 & 13 \\
PCB small & 1 & 1.9 & 284.66 \\
Total & & &
\end{tabular}

The array of sensors are responsible for collecting passive environment data, whereas the capacitive touch screen enables the collection of occupant discomfort. Here occupant discomfort is a user defined quantity on a scale from -3 to $3,-3 / 3$ being the extreme level of too cold/hot, respectively.

The transference of comfort information is occupant enabled by a software defined "update" button on the capacitive touch screen. Since data concerning the occupant is only sent when the occupant dictates, the occupant is in complete control of the release of his/her information. This results in a unique situation in where the frequency of updates by an occupant plays a role in the significance of the updates. For example, if an occupant updates every 10 minutes, each update will carry less weight. Is he updating just to update? Or does he really feel comfortable/uncomfortable as he described? On the other hand if an occupant updates every hour it is more likely that he is responding to changes in comfort, which is a cue to update as a result. It is expected though that occupants will not update very frequently, as they are busy with other stuff. This is why we added a motion sensor. The motion sensor will keep the touch screen on if they are present and turn the touch screen on when their presence is rediscovered. The expectation is that the light of the touch screen turning on will cue the occupant to update. 
The radio and antenna enable the transfer of the recorded occupant and environmental data off of the PCN device and to the base station as seen in Figure 2. The base station consists of a Raspberry Pi and Arduino configuration. From the base station, data gets sent to the database server. More details concerning the base station, database, and multi-PCN networks will be reported in future work.

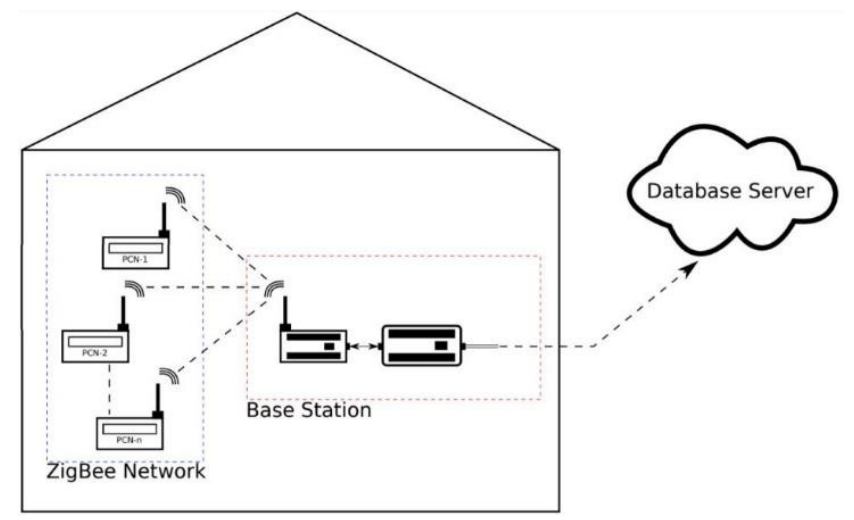

Figure 2. Illustration of multiple PCNs sending data to the base station. The base station relays the data to the database server.

The array of sensors is included so that in the future "comfort models" can be identified. That is, a mapping between the environmental sensor data and the recorded occupant discomfort level. This model would allow for occupant discomfort to be predicted by the environmental data, and thus fully automatic comfort control.

The brain of each PCN device is the Arduino Mega 2560, which was chosen so as to adequately support the devices attached to it (sensors and touchscreen). Numerous libraries are required to support the capacitive touch screen so lighter options such as the Arduino Uno would not work since it has less memory capacity. Additionally, the Arduino Mega also has more I/O pins, so the design is robust to future demands of more sensors.

\section{PCN Device Internal Connections}

The circuit diagram for the connections, created using the Fritzing ${ }^{\oplus}$ software are shown in Figure 3. A printed circuit board (PCB) was fabricated, using the Fritzing ${ }^{\odot}$ schematics, to simplify the assembly and soldering of circuit components, such as external resistors and sensors, and to reduce jumper wires between the components and the Arduino. 


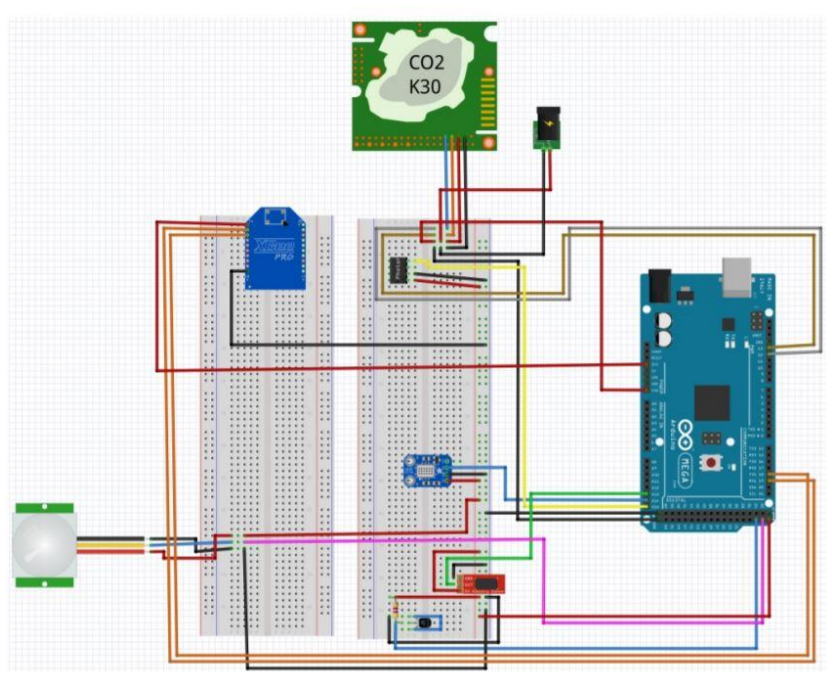

Figure 3. Circuit diagram of a PCN device.

\section{Lessons Learned in Design}

During the design of the device, several problems were encountered that required design reiterations and further testing.

The sensors were outputting data that was incorrect compared to when they were used by themselves with nothing else connected to the Arduino. It was quickly discovered that the supply voltage from the Arduino was lower when all the components were connected. This voltage drop caused some sensors to produce readings that were biased. If the voltage drops then the reading drops proportionally to it. Other sensors responded to the voltage drop with error outputs like constant negative numbers, to indicate something was wrong. The cause of the voltage drop was the touch screen. The touchscreen drew considerably more current than the rest of the components, and the combination was more than the standard power supply could handle, thus causing a voltage drop. The remedy was to use a power supply that could handle more current. A 9V 1A DC power supply was chosen as a result.

Another problem encountered was in unwanted heat. The inside of the case was getting heated due to the heat dissipated by the touchscreen. The design of the enclosure was changed to (i) dissipate heat to the outside more effectively and (ii) shield the sensors from the touchscreen's heat. Additionally, a sleep mode was created for the touch screen using a motion sensor. If the motion sensor detected no motion in the room after 10 minutes, the touch screen display would power off until motion was detected. Further testing indicated these design changes resolved the issue. 


\section{Evaluating the PCN Platform}

\section{Sensor Characterization}

Since most of the sensors used in the PCN device were low-cost sensors, a series of tests were performed to assess their accuracy. This was done before integrating them into the PCN device. A second test was done after the sensors were integrated into the PCN device, to check that variations in power supply, or the presence of the enclosure, or a myriad of other unpredictable factors, reduced their accuracy and consistency. The effects of heat were observed most closely during this test.

characterization of sensors prior to integration with device. This section is to report individual characterization of the sensors before they were integrated into the PCN device. The ground truth for the temperature and humidity sensors was a Vaisala HM70 hand held temperature and humidity sensor with an HMP75 probe. This Vaisala sensor is a highly accurate sensor that is calibrated to deliver accurate measurements of both temperature and humidity. At $20^{\circ} \mathrm{C}$ the accuracy of the temperature sensor is $\pm 0.2^{\circ} \mathrm{C}$. At $20^{\circ} \mathrm{C}$ and within a relative humidity range of 0 to $90 \%$ the accuracy of the humidity sensor is $\pm 1 \% \mathrm{RH}$.
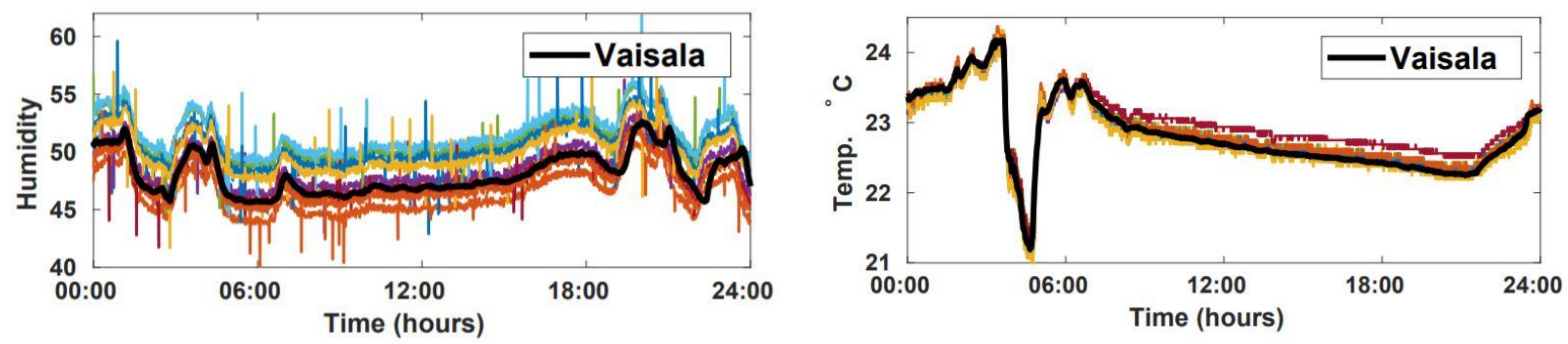

Figure 4. Sensor characterization for ten of both humidity (left) and temperature (right) sensors, compared to one Vaisala sensor.

The results for the temperature and humidity sensor characterization are shown in Figure 4. These figures show the measurements obtained from ten sensors that will be placed on the PCN device and one Vaisala sensor. The temperature sensors are quite accurate, however there appears to be some bias among the humidity sensors. The range of the 10 temperature/humidity sensors was taken at each point in time. The maximum range during the 24 hour period for the temperature sensors was $1.13^{\circ} \mathrm{C}$. The average range was $0.49^{\circ}$, which was considered to be fairly accurate given their price. The humidity sensors had a maximum range of $11.83 \% \mathrm{RH}$ and an average range of $5.55 \% \mathrm{RH}$. Such a high range is due to spikes in readings. These spikes are 
noise as they do not appear in the Vaisala sensor. The bias among the humidity sensors (not a result of noise) falls within $\pm 5 \% \mathrm{RH}$ in relation to the Vaisala sensor. This was a result of cheap sensors and should be taken into account when analyzing data. This was also expected.

Datasheets of the sparkfun HIH4030 humidity sensors show that the accuracy lies within $\pm 3.5 \%$ $\mathrm{RH}$ at $25^{\circ} \mathrm{C}$.

heating assessment. Recall from The PCN device sections that the touchscreen equipment, when powered on for long periods of time, was causing the PCN device to heat up.

Two temperature sensors that were close in readings to each other were used for the heating assessment. That is, the difference between the readings of the two sensors at room temperature conditions and outside of the device was low. The temperature sensors took readings inside a fully installed PCN device. The PCN devices were given an hour to warm up prior to collecting data, so that the bias caused by heat over time could be seen.

The results, as seen in Figure 5, show that the newer model that implemented the excess heat precautions read lower temperatures than before. They also show that the newer model maintained temperature readings at room temperature.

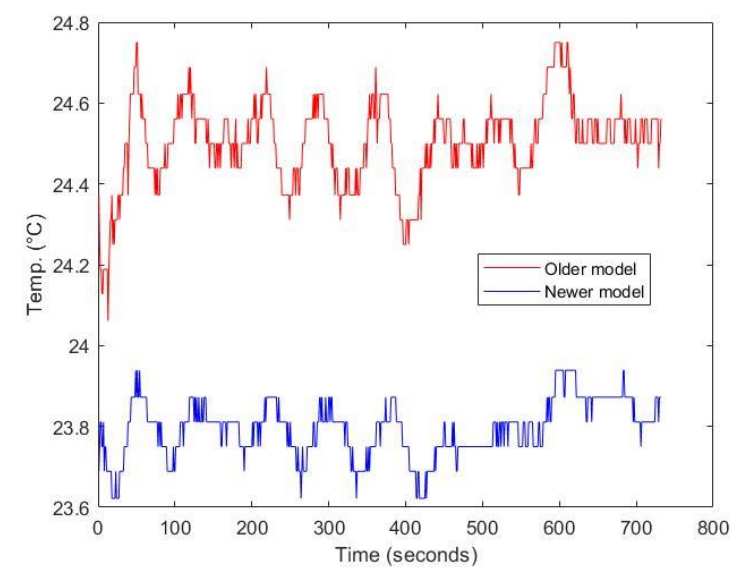

Figure 5. Comparison of temperature readings in PCN device without heat reduction vs temperature readings in PCN with heat reduction.

\section{Wireless Range Test}

The purpose of this test is to validate the network 's communication range. The test is a range test with a single PCN device sending data to the base station reciever. The devices contain only the necessary components of the PCN device and base station that facilitate communication. 


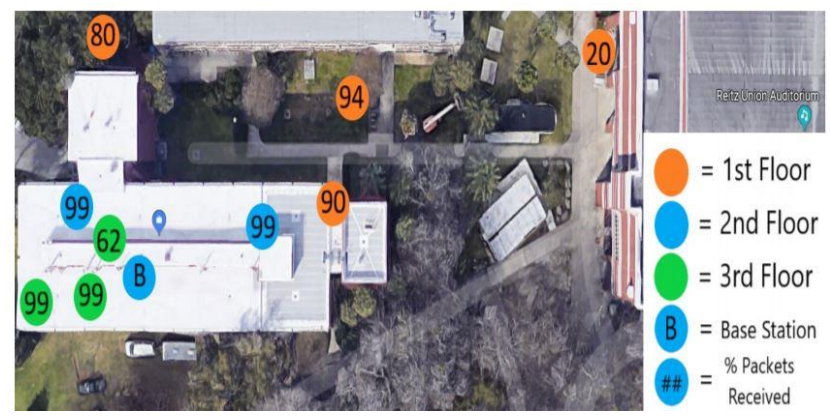

Figure 6. Range test results for a single PCN device. The aim of the test is for the PCN device to send data to the base station (blue circle with B inside).

The results for the range test with the single PCN device are shown in Figure 6. Data transmission on the same floor as the base station was nearly $100 \%$ successful. Between floors, the data transmission success rate started to dwindle. One remedy to this is to create a multi-node mesh network in which data can bounce from node to node until it eventually reaches the base station, but this will be reported in much more detail in future work. Remarkably, the base station is still able to receive some amount of packets even when the PCN device is far outside the test building.

\section{Conclusion}

The PCN device enables occupants to provide feedback on their current comfort level for intelligent building control. The main contribution of the platform - over prior work such as (Gupta et al., 2016) - is wireless connectivity that will enable real time building control. Building occupants interact with their own individual PCN device. The PCN device is equipped with a capacitive touch screen, so interacting with one is similar to that of a smart phone. To provide a sense of empowerment to the occupant, transference of occupant data from the PCN device is dictated by the occupant.

The prototype development described here is a success since it met all the targets, including the below $\$ 300$ per unit cost target. The PCN was evaluated with a string of tests to ensure feasibility of the design. Test results show that the given components can provide reliable data for the comfort model despite the sensors being inexpensive.

Additional work is ongoing on testing and deploying a network of several dozens of devices connecting to one base station. Upon deployment of the devices data will be collected and modeled to develop a control system that controls the HVAC output of the building. All of this work will be reported in future papers. 


\section{Acknowledgements}

I would like to first thank my advisor, Prabir Barooah, for providing me with his guidance and support. I would also like to thank Austin and Ninad for assisting me in my research when I needed it the most. I'd also like to thank Lucas for teaching me everything I needed to know for this project. This project is as much theirs as it is mine. I would also like to thank the University Scholars Program for funding this research. Finally, I would like to thank my family for always being there for me and supporting me.

\section{References}

Ali, A. S., Zanzinger, Z., Debose, D., \& Stephens, B. (2016). Open source building science sensors (osbss): A low-cost arduino-based platform for long-term indoor environmental data collection. Building and Environment, 100, 114 - 126. https://doi.org/10.1016/j.buildenv.2016.02.010

Feldmeier, M., \& Paradiso, J. A. (2010). Personalized hvac control system. Internet of Things 2010 doi: http://doi.org/10.1109/IOT.2010.5678444

Gupta, S. K., Atkingson, S., O’Boyle, I., Drogo, J., Kar, K., Mishra, S., \& Wen, J. T. (2016). Bees: Realtime occupant feedback and environmental learning framework for collaborative thermal management in multi-zone, multi-occupant buildings. Energy and Buildings, 125, 142-152. doi:https://doi.org/10.1016/j.enbuild.2016.04.084.

Jazizadeh, F, Ghahramani, A., Becerik-Gerber, B., Kichkaylo, T., \& Orosz, M. (2014). User-led decentralized thermal comfort driven hvac operations for improved efficiency in office buildings. Energy and Buildings, 70, 398 - 410. doi: https://doi.org/10.1016/j.enbuild.2013.11.066

Pitt, L., Green, P. R., \& Lennox, B. (2013). A sensor network for predicting and maintaining occupant comfort. 2013 IEEE Workshop on Environmental Energy and Structural Monitoring Systems, 16. doi: https://doi.org/10.1109/EESMS.2013.6661696 